\title{
Article \\ New Sufficient Conditions to Ulam Stabilities for a Class of Higher Order Integro-Differential Equations
}

\author{
Alberto M. Simões ${ }^{1,2,3, *,+}+\mathbb{D}$, Fernando Carapau ${ }^{4,5,+} \mathbb{D}$ and Paulo Correia $4,5,+(\mathbb{D}$ \\ 1 CMA-UBI, University of Beira-Interior, 6201-001 Covilhã, Portugal \\ 2 The Center for Research \& Development in Mathematics and Applications (CIDMA), University of Aveiro, \\ 3810-193 Aveiro, Portugal \\ 3 Department of Mathematics, University of Beira Interior, 6201-001 Covilhã, Portugal \\ 4 Centro de Investigação em Matemática e Aplicações (CIMA), University of Évora, 7000-671 Évora, Portugal; \\ flc@uevora.pt (F.C.); pcorreia@uevora.pt (P.C.) \\ 5 Department of Mathematics, University of Évora, 7000-671 Évora, Portugal \\ * Correspondence: asimoes@ubi.pt \\ + These authors contributed equally to this work.
}

check for updates

Citation: Simões, A.M.; Carapau, F.; Correia, P. New Sufficient Conditions to Ulam Stabilities for a Class of Higher Order Integro-Differential Equations. Symmetry 2021, 13, 2068 https://doi.org/10.3390/sym13112068

Academic Editor: José Carlos R. Alcantud

Received: 28 September 2021

Accepted: 26 October 2021

Published: 2 November 2021

Publisher's Note: MDPI stays neutral with regard to jurisdictional claims in published maps and institutional affiliations.

Copyright: (C) 2021 by the authors. Licensee MDPI, Basel, Switzerland. This article is an open access article distributed under the terms and conditions of the Creative Commons Attribution (CC BY) license (https:// creativecommons.org/licenses/by/ $4.0 /)$.
Abstract: In this work, we present sufficient conditions in order to establish different types of Ulam stabilities for a class of higher order integro-differential equations. In particular, we consider a new kind of stability, the $\sigma$-semi-Hyers-Ulam stability, which is in some sense between the Hyers-Ulam and the Hyers-Ulam-Rassias stabilities. These new sufficient conditions result from the application of the Banach Fixed Point Theorem, and by applying a specific generalization of the Bielecki metric.

Keywords: Hyers-Ulam stability; Hyers-Ulam-Rassias stability; Banach fixed point theorem

\section{Introduction}

In 1940, S. M. Ulam [1] proposed the well-known Ulam stability problem. The difficulty of this problem lies in the conditions to be imposed to guarantee the existence of a linear mapping near an approximately linear mapping. It is known that most of the time it is not possible to obtain exact solutions for some integro-differential equations. Therefore, special techniques are applied, allowing us to obtain approximate solutions. In this case, it is crucial to find error bounds to the approximations when replacing the exact solutions in practical problems.

In 1941, D. H. Hyers [2] gave a partial answer to the problem under the assumption that the groups are Banach spaces, considering the additive Cauchy equation $f(x+y)=$ $f(x)+f(y)$. This contribution originated the naming Hyers-Ulam stability. Meanwhile some other approaches came to light, and later in 1978, new directions were introduced by Th. M. Rassias [3] aiming to solve the Ulam stability problem, which gave origin to the concept of Hyers-Ulam-Rassias stability. Furthermore, new developments were carried out involving different norms and other types of equations. We refer in particular to the works presented by T. Aoki [4], Z. Gajda [5] and Th. M. Rassias [6]. For more details on the subject, we refer to $[7,8]$ and the references therein.

The work initiated by S. M. Ulam in the 1940s had relevant consequences in the field of applications as, for example, in chemical reactions, elasticity, fluid flows, semiconductors and population dynamics (see [9-12]). The study of problems involving differential, functional, integro-differential and integral equations, in particular their stability issues, has suffered greatly from the growing engagement over the years with a spread of interest among researchers, for example, see [2,13-27]. Their applicability in mathematical models for which we cannot easily find exact solutions, namely those involving symmetry issues, the study of the stability of the approximate solutions is an open field of research. In particular, this work may be relevant in the study of the stability of the symmetrical flow 
of Newtonian and non-Newtonian fluids related to one-dimensional models obtained by Cosserat Theory associated with fluid dynamics (see $[28,29])$.

\section{Notations and Preliminaries}

Let us consider the higher order integro-differential equation defined by

$$
F\left(x, \varphi(x), \int_{a}^{x} G\left(x, t, \varphi(t), \varphi^{\prime}(t), \ldots, \varphi^{(n-1)}(t)\right) d t\right)=\varphi^{(n)}(x),
$$

with initial conditions

$$
\varphi^{(j)}(a)=0, j=0, \ldots, n-1,
$$

where $n \in \mathbb{N}, \varphi \in C^{n}([a, b])$ and $x \in[a, b]$, with fixed real numbers $a$ and $b$. Moreover, we consider two continuous functions $F:[a, b] \times \mathbb{C} \times \mathbb{C} \rightarrow \mathbb{C}$ and $G:[a, b] \times[a, b] \times \mathbb{C}^{n} \rightarrow \mathbb{C}$.

Next, we introduce several stability concepts related to problem (1) and (2).

Definition 1 (Hyers-Ulam stability). If for each continuously differentiable function $\varphi$ satisfying

$$
\left|F\left(x, \varphi(x), \int_{a}^{x} G\left(x, t, \varphi(t), \varphi^{\prime}(t), \ldots, \varphi^{(n-1)}(t)\right) d t\right)-\varphi^{(n)}(x)\right| \leq \beta,
$$

with $x \in[a, b]$ and $\beta \geq 0$, the higher order integro-differential equation has a solution $\varphi_{0}$ and there is a constant $C>0$, independent of $\varphi_{0}$ and $\varphi$, such that

$$
\left|\varphi_{0}(x)-\varphi(x)\right| \leq C \beta
$$

for all $x \in[a, b]$, then we say that the given problem (1) and (2) has the Hyers-Ulam stability.

Definition 2 (Hyers-Ulam-Rassias stability). Let $\theta$ be a non-decreasing continuous function defined in the interval $[a, b]$. If for each continuously differentiable function $\varphi$ satisfying

$$
\left|F\left(x, \varphi(x), \int_{a}^{x} G\left(x, t, \varphi(t), \varphi^{\prime}(t), \ldots, \varphi^{(n-1)}(t)\right) d t\right)-\varphi^{(n)}(x)\right| \leq \theta(x),
$$

with $x \in[a, b]$, the higher order integro-differential equation has a solution $\varphi_{0}$ and there is $a$ constant $C>0$, independent of $\varphi_{0}$ and $\varphi$, such that

$$
\left|\varphi_{0}(x)-\varphi(x)\right| \leq C \theta(x)
$$

for all $x \in[a, b]$, then we say that the given problem (1) and (2) has the Hyers-Ulam-Rassias stability.

Now, we will introduce a new kind of stability which was presented in [30].

Definition 3 ( $\theta$-semi-Hyers-Ulam stability). Let $\theta$ be a non-decreasing continuous function defined in the interval $[a, b]$. If for each continuously differentiable function $\varphi$ satisfying

$$
\left|F\left(x, \varphi(x), \int_{a}^{x} G\left(x, t, \varphi(t), \varphi^{\prime}(t), \ldots, \varphi^{(n-1)}(t)\right) d t\right)-\varphi^{(n)}(x)\right| \leq \beta,
$$

where $x \in[a, b]$ and $\beta \geq 0$, the higher order integro-differential equation has a solution $\varphi_{0}$ and there is a constant $C>0$, independent of $\varphi_{0}$ and $\varphi$, such that

$$
\left|\varphi_{0}(x)-\varphi(x)\right| \leq C \theta(x)
$$

for all $x \in[a, b]$, then we say that the given problem (1) and (2) has the $\theta$-semi-Hyers-Ulam stability. 
In general, to study the stability of functional, integral and integro-differential equations, it is usual to consider fixed point arguments, for example, see [18,31-38]. Here, we consider the Banach fixed point theorem, which we recall next.

Theorem 1 (Banach fixed point theorem). Let $(X, d)$ be a generalized complete metric space and let $T: X \rightarrow X$ a strictly contractive operator with a Lipschitz constant $L<1$. If there exists a non-negative integer $k$ such that $d\left(T^{k+1} x, T^{k} x\right)<\infty$ for some $x \in X$, then the following three propositions hold true:

1. the sequence $\left(T^{n} x\right)_{n \in \mathbb{N}}$ converges to a fixed point $x^{*}$ of $T$;

2. $x^{*}$ is the unique fixed point of $T$ in $X^{*}=\left\{y \in X: d\left(T^{k} x, y\right)<\infty\right\}$;

3. if $y \in X^{*}$, then

$$
d\left(y, x^{*}\right) \leq \frac{1}{1-L} d(T y, y) .
$$

In the following, we consider the space of continuously differentiable functions in the interval $[a, b], C^{n}([a, b])$, endowed with a generalization of the Bielecki metric, given by

$$
d(\varphi, \psi)=\sup _{x \in[a, b]} \frac{|\varphi(x)-\psi(x)|}{\theta(x)}
$$

with $\theta$ a non-decreasing continuous function $\theta:[a, b] \rightarrow(0, \infty)$ and $\left(C^{n}([a, b]), d\right)$ is a complete metric space (see $[39,40])$.

\section{Hyers-Ulam-Rassias Stability}

In the following theorem we will present sufficient conditions for the Hyers-UlamRassias stability relating to problem (1) and (2).

Theorem 2. Let $\theta:[a, b] \rightarrow(0, \infty)$ be a non-decreasing continuous function which fulfills the inequality

$$
\int_{a}^{x} \theta(t) d t \leq \alpha \theta(x),
$$

for all $x \in[a, b]$, where $\alpha \in \mathbb{R}$. Suppose also that the continuous function $F:[a, b] \times \mathbb{C} \times \mathbb{C} \rightarrow \mathbb{C}$ satisfies the condition

$$
|F(x, u(x), g(x))-F(x, v(x), h(x))| \leq M(|u(x)-v(x)|+|g(x)-h(x)|)
$$

where $M>0$ is a Lipschitz constant, and the continuous kernel $G:[a, b] \times[a, b] \times \mathbb{C}^{n} \rightarrow \mathbb{C}$ satisfies the condition

$$
\left|G\left(x, t, u(t), u^{\prime}(t), \ldots, u^{(n-1)}(t)\right)-G\left(x, t, v(t), v^{\prime}(t), \ldots, v^{(n-1)}(t)\right)\right| \leq L|u(t)-v(t)|
$$

where $L>0$ is a Lipschitz constant. If $\varphi \in C^{n}([a, b])$ is such that

$$
\left|F\left(x, \varphi(x), \int_{a}^{x} G\left(x, t, \varphi(t), \varphi^{\prime}(t), \ldots, \varphi^{(n-1)}(t)\right) d t\right)-\varphi^{(n)}(x)\right| \leq \theta(x),
$$

where $x \in[a, b]$, and $M\left(\alpha^{n}+L \alpha^{n+1}\right)<1$, then there is a unique function $\varphi_{0} \in C^{n}([a, b])$, solution of problem (1) and (2), such that

$$
\left|\varphi_{0}(x)-\varphi(x)\right| \leq \frac{\alpha^{n}}{1-M\left(\alpha^{n}+L \alpha^{n+1}\right)} \theta(x),
$$

for all $x \in[a, b]$. 
Proof. Considering the initial conditions (2), i.e., $\varphi^{(j)}(a)=0, j=0, \ldots, n-1$ and using integration properties, Equation (1) is equivalent to

$$
\begin{aligned}
\int_{a}^{x} \int_{a}^{r_{n}} \int_{a}^{r_{n-1}} \ldots \int_{a}^{r_{2}} F\left(\left(r_{1}, \varphi\left(r_{1}\right),\right.\right. & \\
& \left.\int_{a}^{r_{1}} G\left(r_{1}, t, \varphi(t), \varphi^{\prime}(t), \ldots, \varphi^{(n-1)}(t)\right) d t\right) d r_{1} \ldots d r_{n-2} d r_{n-1} d r_{n}=\varphi(x) .
\end{aligned}
$$

Let us define the continuous operator $T: C^{n}([a, b]) \rightarrow C^{n}([a, b])$ by

$$
\begin{aligned}
(T \varphi)(x)=\int_{a}^{x} & \int_{a}^{r_{n}} \int_{a}^{r_{n-1}} \ldots \int_{a}^{r_{2}} F\left(r_{1}, \varphi\left(r_{1}\right),\right. \\
& \left.\int_{a}^{r_{1}} G\left(r_{1}, t, \varphi(t), \varphi^{\prime}(t), \ldots, \varphi^{(n-1)}(t)\right) d t\right) d r_{1} \ldots d r_{n-2} d r_{n-1} d r_{n},
\end{aligned}
$$

for all $x \in[a, b]$ and $\varphi \in C^{n}([a, b])$. Indeed, for any continuous function $\varphi$, we have

$$
\begin{aligned}
\left|(T \varphi)(x)-(T \varphi)\left(x_{0}\right)\right|=\mid \int_{a}^{x} \int_{a}^{r_{n}} \int_{a}^{r_{n-1}} \ldots \int_{a}^{r_{2}} F\left(r_{1}, \varphi\left(r_{1}\right),\right. \\
\left.\quad \int_{a}^{r_{1}} G\left(r_{1}, t, \varphi(t), \varphi^{\prime}(t), \ldots, \varphi^{(n-1)}(t)\right) d t\right) d r_{1} \ldots d r_{n-2} d r_{n-1} d r_{n} \\
\quad-\int_{a}^{x_{0}} \int_{a}^{r_{n}} \int_{a}^{r_{n-1}} \ldots \int_{a}^{r_{2}} F\left(r_{1}, \varphi\left(r_{1}\right),\right. \\
\left.\quad \int_{a}^{r_{1}} G\left(r_{1}, t, \varphi(t), \varphi^{\prime}(t), \ldots, \varphi^{(n-1)}(t)\right) d t\right) d r_{1} \ldots d r_{n-2} d r_{n-1} d r_{n} \mid \\
=\mid \int_{x}^{x_{0}} \int_{a}^{r_{n}} \int_{a}^{r_{n-1}} \ldots \int_{a}^{r_{2}} F\left(r_{1}, \varphi\left(r_{1}\right),\right. \\
\left.\quad \int_{a}^{r_{1}} G\left(r_{1}, t, \varphi(t), \varphi^{\prime}(t), \ldots, \varphi^{(n-1)}(t)\right) d t\right) d r_{1} \ldots d r_{n-2} d r_{n-1} d r_{n} \mid \longrightarrow 0
\end{aligned}
$$

when $x \rightarrow x_{0}$. Therefore, using condition (11), we have

$$
\begin{aligned}
\int_{a}^{x} \int_{a}^{r_{n}} \int_{a}^{r_{n-1}} & \ldots \int_{a}^{r_{2}} \int_{a}^{r_{1}} \theta(t) d t d r_{1} \ldots d r_{n-2} d r_{n-1} d r_{n} \\
& \leq \int_{a}^{x} \int_{a}^{r_{n}} \int_{a}^{r_{n-1}} \ldots \int_{a}^{r_{2}} \alpha \theta\left(r_{1}\right) d r_{1} \ldots d r_{n-2} d r_{n-1} d r_{n} \\
& \leq \int_{a}^{x} \int_{a}^{r_{n}} \int_{a}^{r_{n-1}} \ldots \int_{a}^{r_{3}} \alpha^{2} \theta\left(r_{2}\right) d r_{2} \ldots d r_{n-2} d r_{n-1} d r_{n} \\
& \vdots \\
& \leq \int_{a}^{x} \alpha^{n} \theta\left(r_{n}\right) d r_{n} \\
& \leq \alpha^{n+1} \theta(x) .
\end{aligned}
$$

Consequently, using conditions (17) and (18), we will prove that the operator $T$ is strictly contractive to the Bielecki metric (10). In order to prove that, we have for all $\varphi, \psi \in C^{n}([a, b]):$

$$
\begin{aligned}
& d(T \varphi, T \psi)=\sup _{x \in[a, b]} \frac{|(T \varphi)(x)-(T \psi)(x)|}{\theta(x)} \\
& =\sup _{x \in[a, b]} \frac{1}{\theta(x)} \mid \int_{a}^{x} \int_{a}^{r_{n}} \int_{a}^{r_{n-1}} \ldots \int_{a}^{r_{2}} F\left(r_{1}, \varphi\left(r_{1}\right), \int_{a}^{r_{1}} G\left(r_{1},\right.\right. \\
& \left.\left.\quad t, \varphi(t), \varphi^{\prime}(t), \ldots, \varphi^{(n-1)}(t)\right) d t\right) d r_{1} \ldots d r_{n-2} d r_{n-1} d r_{n}
\end{aligned}
$$




$$
\begin{aligned}
& -\int_{a}^{x} \int_{a}^{r_{n}} \int_{a}^{r_{n-1}} \cdots \int_{a}^{r_{2}} F\left(r_{1}, \psi\left(r_{1}\right), \int_{a}^{r_{1}} G\left(r_{1},\right.\right. \\
& \left.\left.t, \psi(t), \psi^{\prime}(t), \ldots, \psi^{(n-1)}(t)\right) d t\right) d r_{1} \ldots d r_{n-2} d r_{n-1} d r_{n} \\
& \leq \sup _{x \in[a, b]} \frac{1}{\theta(x)} \int_{a}^{x} \int_{a}^{r_{n}} \int_{a}^{r_{n-1}} \cdots \int_{a}^{r_{2}} \mid F\left(r_{1}\right. \\
& \left.\varphi\left(r_{1}\right), \int_{a}^{r_{1}} G\left(r_{1}, t, \varphi(t), \varphi^{\prime}(t), \ldots, \varphi^{(n-1)}(t)\right) d t\right) \\
& -F\left(r_{1}, \psi\left(r_{1}\right), \int_{a}^{r_{1}} G\left(r_{1}, t, \psi(t),\right.\right. \\
& \left.\left.\psi^{\prime}(t), \ldots, \psi^{(n-1)}(t)\right) d t\right) \mid d r_{1} \ldots d r_{n-2} d r_{n-1} d r_{n} \\
& \leq M \sup _{x \in[a, b]} \frac{1}{\theta(x)} \int_{a}^{x} \int_{a}^{r_{n}} \int_{a}^{r_{n-1}} \ldots \int_{a}^{r_{2}}\left|\varphi\left(r_{1}\right)-\psi\left(r_{1}\right)\right| d r_{1} \ldots d r_{n-2} d r_{n-1} d r_{n} \\
& +M \sup _{x \in[a, b]} \frac{1}{\theta(x)} \int_{a}^{x} \int_{a}^{r_{n}} \int_{a}^{r_{n}-1} \cdots \int_{a}^{r_{2}} \int_{a}^{r_{1}} \mid G\left(r_{1}, t, \varphi(t), \varphi^{\prime}(t), \ldots, \varphi^{(n-1)}(t)\right) \\
& -G\left(r_{1}, t, \psi(t), \psi^{\prime}(t), \ldots, \psi^{(n-1)}(t)\right) \mid d t d r_{1} \ldots d r_{n-2} d r_{n-1} d r_{n} \\
& \leq M \sup _{x \in[a, b]} \frac{1}{\theta(x)} \int_{a}^{x} \int_{a}^{r_{n}} \int_{a}^{r_{n-1}} \ldots \int_{a}^{r_{2}}\left|\varphi\left(r_{1}\right)-\psi\left(r_{1}\right)\right| d r_{1} \ldots d r_{n-2} d r_{n-1} d r_{n} \\
& +M L \sup _{x \in[a, b]} \frac{1}{\theta(x)} \int_{a}^{x} \int_{a}^{r_{n}} \int_{a}^{r_{n-1}} \cdots \int_{a}^{r_{2}} \\
& \int_{a}^{r_{1}}|\varphi(t)-\psi(t)| d t d r_{1} \ldots d r_{n-2} d r_{n-1} d r_{n} \\
& =M \sup _{x \in[a, b]} \frac{1}{\theta(x)} \int_{a}^{x} \int_{a}^{r_{n}} \int_{a}^{r_{n-1}} \ldots \int_{a}^{r_{2}} \theta\left(r_{1}\right) \frac{\left|\varphi\left(r_{1}\right)-\psi\left(r_{1}\right)\right|}{\theta\left(r_{1}\right)} d r_{1} \ldots d r_{n-2} d r_{n-1} d r_{n} \\
& +M L \sup _{x \in[a, b]} \frac{1}{\theta(x)} \int_{a}^{x} \int_{a}^{r_{n}} \int_{a}^{r_{n}-1} \cdots \int_{a}^{r_{2}} \\
& \int_{a}^{r_{1}} \theta(t) \frac{|\varphi(t)-\psi(t)|}{\theta(t)} d t d r_{1} \ldots d r_{n-2} d r_{n-1} d r_{n} \\
& \leq M \sup _{r_{1} \in[a, b]} \frac{\left|\varphi\left(r_{1}\right)-\psi\left(r_{1}\right)\right|}{\theta\left(r_{1}\right)} \sup _{x \in[a, b]} \frac{1}{\theta(x)} \int_{a}^{x} \int_{a}^{r_{n}} \\
& \int_{a}^{r_{n-1}} \cdots \int_{a}^{r_{2}} \theta\left(r_{1}\right) d r_{1} \ldots d r_{n-2} d r_{n-1} d r_{n} \\
& +M L \sup _{t \in[a, b]} \frac{|\varphi(t)-\psi(t)|}{\theta(t)} \sup _{x \in[a, b]} \frac{1}{\theta(x)} \int_{a}^{x} \int_{a}^{r_{n}} \\
& \int_{a}^{r_{n-1}} \cdots \int_{a}^{r_{2}} \int_{a}^{r_{1}} \theta(t) d t d r_{1} \ldots d r_{n-2} d r_{n-1} d r_{n} \\
& \leq \operatorname{Md}(\varphi, \psi) \alpha^{n}+\operatorname{MLd}(\varphi, \psi) \sup _{x \in[a, b]} \frac{\alpha^{n+1} \theta(x)}{\theta(x)} \\
& =M\left(\alpha^{n}+L \alpha^{n+1}\right) d(\varphi, \psi) .
\end{aligned}
$$

Therefore, by the fact that

$$
M\left(\alpha^{n}+L \alpha^{n+1}\right)<1
$$


we have that the operator $T$ is strictly contractive. Thus, we can apply Theorem 1 , which ensures that we have the Hyers-Ulam-Rassias stability for problem (1) and (2). Additionally, from (14), we have

$$
-\theta(x) \leq F\left(x, \varphi(x), \int_{a}^{x} G\left(x, t, \varphi(t), \varphi^{\prime}(t), \ldots, \varphi^{(n-1)}(t)\right) d t\right)-\varphi^{(n)}(x) \leq \theta(x),
$$

where $x \in[a, b]$. Therefore, using integration, we obtain

$$
\begin{aligned}
& \mid \int_{a}^{x} \int_{a}^{r_{n}} \int_{a}^{r_{n-1}} \ldots \int_{a}^{r_{2}} F\left(r_{1}, \varphi\left(r_{1}\right), \int_{a}^{r_{1}} G\left(r_{1},\right.\right. \\
& \left.\left.\quad t, \varphi(t), \varphi^{\prime}(t), \ldots, \varphi^{(n-1)}(t)\right) d t\right) d r_{1} \ldots d r_{n-2} d r_{n-1} d r_{n}-\varphi(x) \mid \\
& \quad \leq \int_{a}^{x} \int_{a}^{r_{n}} \int_{a}^{r_{n-1}} \ldots \int_{a}^{r_{2}} \theta\left(r_{1}\right) d r_{1} \ldots d r_{n-2} d r_{n-1} d r_{n} \\
& \quad \leq \alpha^{n} \theta(x) .
\end{aligned}
$$

Now, using conditions (16) and (20), we have

$$
|(T \varphi)(x)-\varphi(x)| \leq \alpha^{n} \theta(x), \quad x \in[a, b] .
$$

Moreover, from (9) follows

$$
d\left(\varphi_{0}, \varphi\right) \leq \frac{1}{1-M\left(\alpha^{n}+L \alpha^{n+1}\right)} d(T \varphi, \varphi) .
$$

Finally, from the definition of the metric $d$ and (21), we obtain

$$
\sup _{x \in[a, b]} \frac{\left|\varphi_{0}(x)-\varphi(x)\right|}{\theta(x)} \leq \frac{\alpha^{n}}{1-M\left(\alpha^{n}+L \alpha^{n+1}\right)}
$$

and consequently condition (15) holds.

\section{4. $\theta$-Semi-Hyers-Ulam and Hyers-Ulam Stabilities}

Next theorems presents sufficient conditions for the $\theta$-semi-Hyers-Ulam stability and for the Hyers-Ulam stability regarding problem (1) and (2).

Theorem 3. Let $\theta:[a, b] \rightarrow(0, \infty)$ be a non-decreasing continuous function which fulfills the inequality

$$
\int_{a}^{x} \theta(t) d t \leq \alpha \theta(x)
$$

for all $x \in[a, b]$, where $\alpha \in \mathbb{R}$. Suppose also that the continuous function $F:[a, b] \times \mathbb{C} \times \mathbb{C} \rightarrow \mathbb{C}$ satisfies the condition

$$
|F(x, u(x), g(x))-F(x, v(x), h(x))| \leq M(|u(x)-v(x)|+|g(x)-h(x)|)
$$

where $M>0$ is a Lipschitz constant, and the continuous kernel $G:[a, b] \times[a, b] \times \mathbb{C}^{n} \rightarrow \mathbb{C}$ satisfies the condition

$$
\left|G\left(x, t, u(t), u^{\prime}(t), \ldots, u^{(n-1)}(t)\right)-G\left(x, t, v(t), v^{\prime}(t), \ldots, v^{(n-1)}(t)\right)\right| \leq L|u(t)-v(t)|
$$

where $L>0$ is a Lipschitz constant. If $\varphi \in C^{n}([a, b])$ is such that

$$
\left|F\left(x, \varphi(x), \int_{a}^{x} G\left(x, t, \varphi(t), \varphi^{\prime}(t), \ldots, \varphi^{(n-1)}(t)\right) d t\right)-\varphi^{(n)}(x)\right| \leq \beta,
$$


where $x \in[a, b], \beta \geq 0$, and $M\left(\alpha^{n}+L \alpha^{n+1}\right)<1$, then there exists a unique function $\varphi_{0} \in$ $C^{n}([a, b])$, solution of the problem (1) and (2), such that

$$
\left|\varphi_{0}(x)-\varphi(x)\right| \leq \frac{(b-a)^{n} \beta}{\left(1-M\left(\alpha^{n}+L \alpha^{n+1}\right)\right) \theta(a)} \theta(x),
$$

for all $x \in[a, b]$.

Proof. Let us consider the operator $T: C^{n}([a, b]) \rightarrow C^{n}([a, b])$, defined by

$$
\begin{aligned}
& (T \varphi)(x)=\int_{a}^{x} \int_{a}^{r_{n}} \int_{a}^{r_{n-1}} \ldots \int_{a}^{r_{2}} F\left(r_{1}, \varphi\left(r_{1}\right),\right. \\
& \left.\int_{a}^{r_{1}} G\left(r_{1}, t, \varphi(t), \varphi^{\prime}(t), \ldots, \varphi^{(n-1)}(t)\right) d t\right) d r_{1} \ldots d r_{n-2} d r_{n-1} d r_{n}
\end{aligned}
$$

for all $x \in[a, b]$ and $\varphi \in C^{n}([a, b])$.

Following the same ideas as in the proof of Theorem 2, we can prove that $T$ is strictly contractive to the metric (10) due to the fact that

$$
M\left(\alpha^{n}+L \alpha^{n+1}\right)<1 .
$$

Therefore, we can apply Theorem 1, which ensures that the $\theta$-semi-Hyers-Ulam stability condition is valid for problem (1) and (2). Moreover, using condition (27) and integration, we obtain

$$
\begin{aligned}
\mid \int_{a}^{x} \int_{a}^{r_{n}} \int_{a}^{r_{n-1}} \ldots \int_{a}^{r_{2}} F\left(r_{1}, \varphi\left(r_{1}\right), \int_{a}^{r_{1}} G\left(r_{1}, t\right.\right. \\
\\
\left.\left.\quad \varphi(t), \varphi^{\prime}(t), \ldots, \varphi^{(n-1)}(t)\right) d t\right) d r_{1} \ldots d r_{n-2} d r_{n-1} d r_{n}-\varphi(x) \mid \\
\quad \leq \int_{a}^{x} \int_{a}^{r_{n}} \int_{a}^{r_{n-1}} \ldots \int_{a}^{r_{2}} \beta d r_{1} \ldots d r_{n-2} d r_{n-1} d r_{n} \\
\quad \leq \beta(b-a)^{n} .
\end{aligned}
$$

Next, using conditions (29) and (30), we obtain

$$
|(T \varphi)(x)-\varphi(x)| \leq \beta(b-a)^{n},
$$

for all $x \in[a, b]$. From (9), Bielecki metric $d$, and (31), follows

$$
\sup _{x \in[a, b]} \frac{\left|\varphi_{0}(x)-\varphi(x)\right|}{\theta(x)} \leq \frac{1}{1-M\left(\alpha^{n}+L \alpha^{n+1}\right)} \sup _{x \in[a, b]} \frac{\beta(b-a)^{n}}{\theta(x)} .
$$

Consequently, considering the definition of $\theta$, the condition (28) holds.

By using Theorem 3, we can deduce the Hyers-Ulam stability to problem (1) and (2).

Theorem 4. Let $\theta:[a, b] \rightarrow(0, \infty)$ be a non-decreasing continuous function, which fulfills the inequality

$$
\int_{a}^{x} \theta(t) d t \leq \alpha \theta(x)
$$

for all $x \in[a, b]$, where $\alpha \in \mathbb{R}$. Suppose also that the continuous function $F:[a, b] \times \mathbb{C} \times \mathbb{C} \rightarrow \mathbb{C}$ satisfies the condition

$$
|F(x, u(x), g(x))-F(x, v(x), h(x))| \leq M(|u(x)-v(x)|+|g(x)-h(x)|)
$$


where $M>0$ is a Lipschitz constant, and the continuous kernel $G:[a, b] \times[a, b] \times \mathbb{C}^{n} \rightarrow \mathbb{C}$ satisfies the condition

$$
\left|G\left(x, t, u(t), u^{\prime}(t), \ldots, u^{(n-1)}(t)\right)-G\left(x, t, v(t), v^{\prime}(t), \ldots, v^{(n-1)}(t)\right)\right| \leq L|u(t)-v(t)|
$$

where $L>0$ is a Lipschitz constant. If $\varphi \in C^{n}([a, b])$ is such that

$$
\left|F\left(x, \varphi(x), \int_{a}^{x} G\left(x, t, \varphi(t), \varphi^{\prime}(t), \ldots, \varphi^{(n-1)}(t)\right) d t\right)-\varphi^{(n)}(x)\right| \leq \beta,
$$

where $x \in[a, b], \beta \geq 0$, and $M\left(\alpha^{n}+L \alpha^{n+1}\right)<1$, then there exists a unique function $\varphi_{0} \in C^{n}([a, b])$, solution of the problem (1)-(2), such that

$$
\left|\varphi_{0}(x)-\varphi(x)\right| \leq \frac{(b-a)^{n} \theta(b)}{\left(1-M\left(\alpha^{n}+L \alpha^{n+1}\right)\right) \theta(a)} \beta,
$$

for all $x \in[a, b]$.

\section{Examples}

In this section, we present some examples in order to illustrate the results obtained throughout the work.

\subsection{First Example: 2-Differentiable Function}

Let us define the space

$$
\mathbb{D}=\left\{u, v \in C^{n}\left(\left[0, \frac{\pi}{2}\right]\right):\left|u^{\prime}(x)-v^{\prime}(x)\right| \leq|u(x)-v(x)|\right\} .
$$

We consider the 2-differentiable functions $\varphi:\left[0, \frac{\pi}{2}\right] \rightarrow \mathbb{R}$ on the space $\mathbb{D}$, and the integro-differential equation given by

$$
-2 \varphi(x)-x \sin (x)-\cos (x)+\frac{x^{2}}{2}+\int_{0}^{x} t \varphi(t)+\varphi^{\prime}(t) d t=\varphi^{\prime \prime}(x),
$$

with $x \in\left[0, \frac{\pi}{2}\right]$.

We also consider the continuous function $\theta:\left[0, \frac{\pi}{2}\right] \rightarrow(0, \infty)$ defined by

$$
\theta(x)=0.1 e^{2.2 x},
$$

which fulfills the inequality

$$
\int_{0}^{x} 0.1 e^{2.2 t} d t \leq \alpha 0.1 e^{2.2 x}=\alpha \theta(x),
$$

with $\alpha \in\left[\frac{10}{22}\left(1-e^{-\frac{22}{20} \pi}\right), \infty\right)$.

Thus, considering these assumptions all the conditions of Theorem 2 are satisfied.

Now, considering the continuous function $F:\left[0, \frac{\pi}{2}\right] \times \mathbb{C} \times \mathbb{C} \rightarrow \mathbb{C}$ defined by

$$
F(x, u(x), g(x))=-2 u(x)-x \sin (x)-\cos (x)+\frac{x^{2}}{2}+g(x),
$$

we have

$$
|F(x, u(x), g(x))-F(x, v(x), h(x))| \leq 2(|u(x)-v(x)|+|g(x)-h(x)|)
$$

with $M=2$. 
Moreover, the kernel $G:\left[0, \frac{\pi}{2}\right] \times\left[0, \frac{\pi}{2}\right] \times \mathbb{C} \rightarrow \mathbb{C}$ defined by

$$
G\left(x, t, u(t), u^{\prime}(t)\right)=t u(t)+u^{\prime}(t)
$$

is a continuous function satisfying

$$
\left|G\left(x, t, u(t), u^{\prime}(t)\right)-G\left(x, t, v(t), v^{\prime}(t)\right)\right| \leq\left(\frac{\pi}{2}+1\right)|u(t)-v(t)|
$$

with $L=\frac{\pi}{2}+1$.

Consequently, by choosing $\varphi(x)=\cos \left(\frac{101}{100} x\right)-\frac{102}{100}$, it follows

$$
\begin{gathered}
\left|\varphi^{\prime \prime}(x)+2 \varphi(x)+x \sin (x)+\cos (x)-\frac{x^{2}}{2}-\int_{0}^{x} t \varphi(t)+\varphi^{\prime}(t) d t\right| \\
=\mid-\frac{102,050,401}{102,010,000} \cos \left(\frac{101}{100} x\right)-\frac{15,226}{255,025}+x \sin (x)+\cos (x) \\
-\frac{49}{100} x^{2}-\frac{100}{101} x \sin \left(\frac{101}{100} x\right) \mid \leq \theta(x),
\end{gathered}
$$

for all $x \in\left[0, \frac{\pi}{2}\right]$. Then, for any

$$
\alpha \in\left[\frac{10}{22}\left(1-e^{-\frac{22}{20} \pi}\right), 0.474575\right),
$$

we have $M\left(\alpha^{2}+L \alpha^{3}\right)<1$.

Therefore, we have the Hyers-Ulam-Rassias stability of the integro-differential Equation (38).

Moreover, if we consider the exact solution $\varphi_{0}(x)=\cos (x)-1$, we obtain

$$
\left|\varphi_{0}(x)-\varphi(x)\right|=\left|\cos (x)-\cos \left(\frac{101}{100} x\right)+\frac{1}{50}\right| \leq \frac{\alpha^{2}}{1-2\left(\alpha^{2}+\left(\frac{\pi}{2}+1\right) \alpha^{3}\right)} 0.1 e^{2.2 x}
$$

for all $x \in\left[0, \frac{\pi}{2}\right]$.

Both inequalities (42) and (43) are illustrated in Figures 1 and 2.
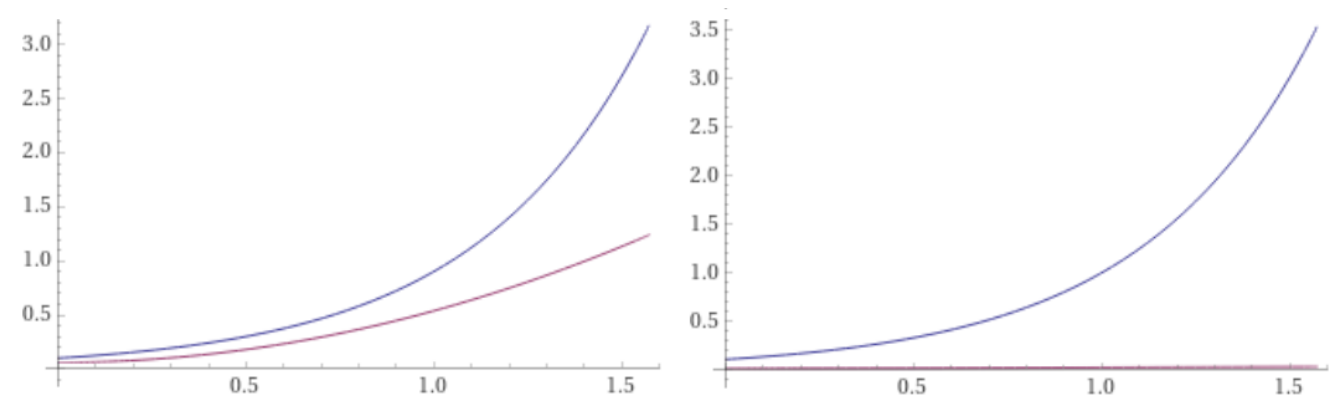

Figure 1. On the left, we have $\theta(x)=0.1 e^{2.2 x}$ and $z_{1}(x)=$ $\left|-\frac{102,050,401}{102,010,000} \cos \left(\frac{101}{100} x\right)-\frac{15,226}{255,025}+x \sin (x)+\cos (x)-\frac{49}{100} x^{2}-\frac{100}{101} x \sin \left(\frac{101}{100} x\right)\right|$ to illustrate the inequality (42). On the right, we have $z_{2}(x)=\frac{\alpha^{2}}{1-2\left(\alpha^{2}+\left(\frac{\pi}{2}+1\right) \alpha^{3}\right)} 0.1 e^{2.2 x}$ with $\alpha=0.4402$ and $z_{3}(x)=\left|\cos (x)-\cos \left(\frac{101}{100} x\right)+\frac{1}{50}\right|$ to illustrate the inequality (43). 

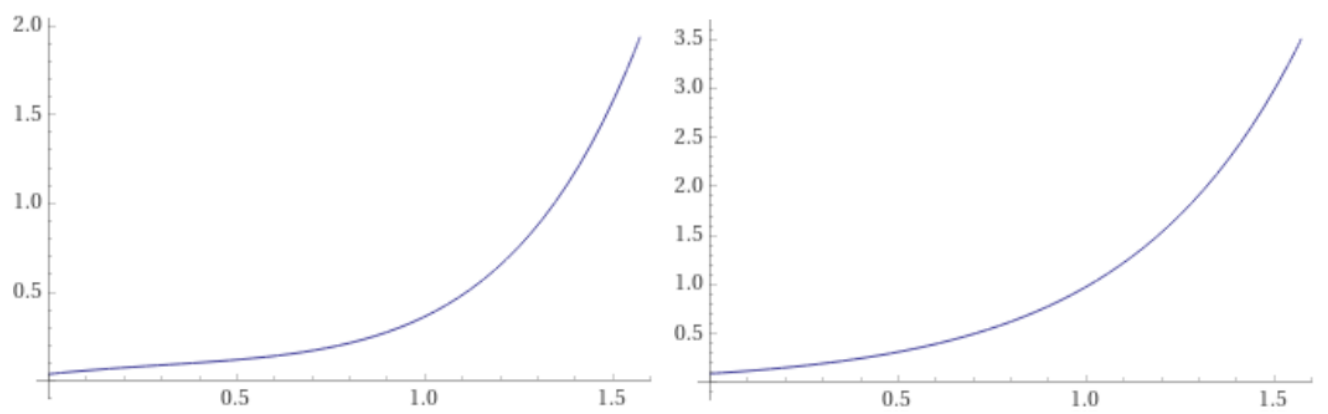

Figure 2. On the left, we have $z_{1}(x)=\theta(x)-\left|\varphi^{\prime \prime}(x)-F\left(x, \varphi(x), \int_{0}^{x} G\left(x, t, \varphi(t), \varphi^{\prime}(t)\right) d t\right)\right|$ to illustrate the inequality (42). On the right, we have $z_{2}(x)=\frac{\alpha^{2}}{1-2\left(\alpha^{2}+\left(\frac{\pi}{2}+1\right) \alpha^{3}\right)} \theta(x)-\left|\varphi_{0}(x)-\varphi(x)\right|$ with $\alpha=0.4402$ to illustrate the inequality (43).

\subsection{Second Example: 3-Differentiable Function}

In this example, we consider the 3-differentiable functions $\varphi:\left[0, \frac{1}{2}\right] \rightarrow \mathbb{R}$, and the integro-differential equation given by

$$
-\frac{1}{6} x^{3} \varphi(x)-\frac{1}{5} x \varphi(x)+24 x+\int_{0}^{x} t \varphi(t)+\varphi(t) d t=\varphi^{\prime \prime \prime}(x),
$$

with $x \in\left[0, \frac{1}{2}\right]$. Moreover, we consider the continuous function $\theta:\left[0, \frac{1}{2}\right] \rightarrow(0, \infty)$ defined by

$$
\theta(x)=3.2 e^{2.1 x}
$$

which fulfills the inequality

$$
\int_{0}^{x} 3.2 e^{2.1 t} d t \leq \alpha 3.2 e^{2.1 x}=\alpha \theta(x)
$$

with $\alpha \in\left[\frac{10}{21}\left(1-e^{-\frac{21}{20}}\right), \infty\right)$. Therefore, all conditions of Theorem 2 are satisfied.

Let us consider the continuous function $F:\left[0, \frac{1}{2}\right] \times \mathbb{C} \times \mathbb{C} \rightarrow \mathbb{C}$ defined by

$$
F(x, u(x), g(x))=-\frac{1}{6} x^{3} u(x)-\frac{1}{5} x u(x)+24 x+g(x),
$$

which verifies

$$
|F(x, u(x), g(x))-F(x, v(x), h(x))| \leq|u(x)-v(x)|+|g(x)-h(x)|
$$

with $M=1$. Furthermore, the kernel $G:\left[0, \frac{1}{2}\right] \times\left[0, \frac{1}{2}\right] \times \mathbb{C} \rightarrow \mathbb{C}$ defined by

$$
G\left(x, t, u(t), u^{\prime}(t)\right)=t u(t)+u(t)
$$

is a continuous function and verifies

$$
\left|G\left(x, t, u(t), u^{\prime}(t)\right)-G\left(x, t, v(t), v^{\prime}(t)\right)\right| \leq \frac{5}{4}|u(t)-v(t)|
$$

with $L=\frac{5}{4}$. Now, if we choose $\varphi(x)=0.53 x^{3}$, it follows

$$
\left|\varphi^{\prime \prime \prime}(x)+\frac{1}{6} x^{3} \varphi(x)+\frac{1}{5} x \varphi(x)-24 x-\int_{0}^{x} t \varphi(t)+\varphi(t) d t\right|=\left|\frac{318}{100}-24 x\right| \leq \theta(x),
$$


for all $x \in\left[0, \frac{1}{2}\right]$. Additionally, for any $\alpha \in\left[\frac{10}{21}\left(1-e^{-\frac{21}{20}}\right), 0.794596\right)$ we have $M\left(\alpha^{3}+\right.$ $\left.L \alpha^{4}\right)<1$. Consequently, we have the Hyers-Ulam-Rassias stability of the integrodifferential Equation (44). If we consider the exact solution $\varphi_{0}(x)=x^{4}$, we obtain

$$
\left|\varphi_{0}(x)-\varphi(x)\right|=\left|0.53 x^{3}-x^{4}\right| \leq \frac{\alpha^{3}}{1-\left(\alpha^{3}+\frac{5}{4} \alpha^{4}\right)} 3.2 e^{2.1 x}
$$

for all $x \in\left[0, \frac{1}{2}\right]$, which is in accordance with the presented results.

Both inequalities (48) and (49) are illustrated in Figures 3 and 4.
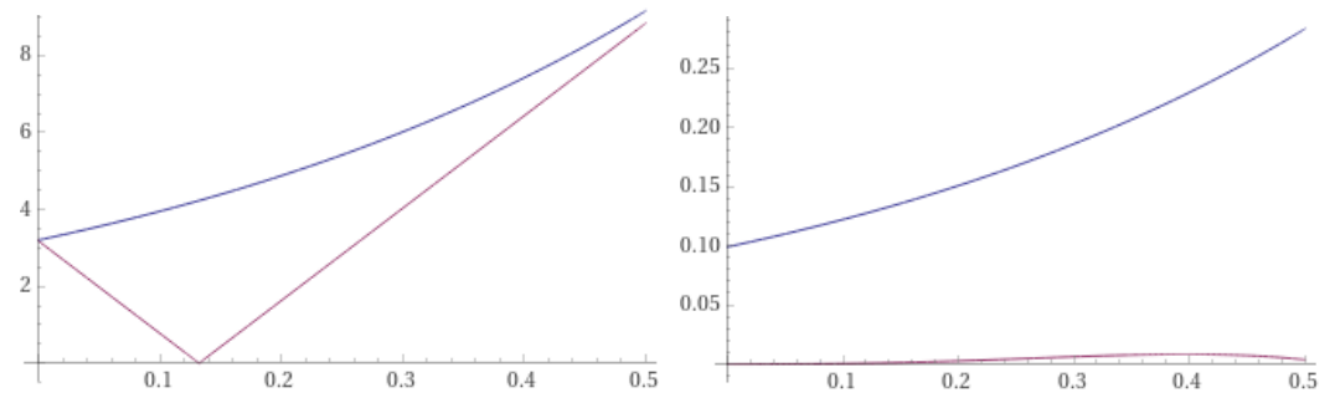

Figure 3. To illustrate the inequality (48), we have on the left $\theta(x)=3.2 e^{2.1 x}$ and $z_{1}(x)=\left|\frac{318}{100}-24 x\right|$. On the right, we have $z_{2}(x)=\frac{\alpha^{3}}{1-\left(\alpha^{3}+\frac{5}{4} \alpha^{4}\right)} 3.2 e^{2.1 x}$ with $\alpha=0.3095535$ and $z_{3}(x)=\left|0.53 x^{3}-x^{4}\right|$ to illustrate the inequality (49).
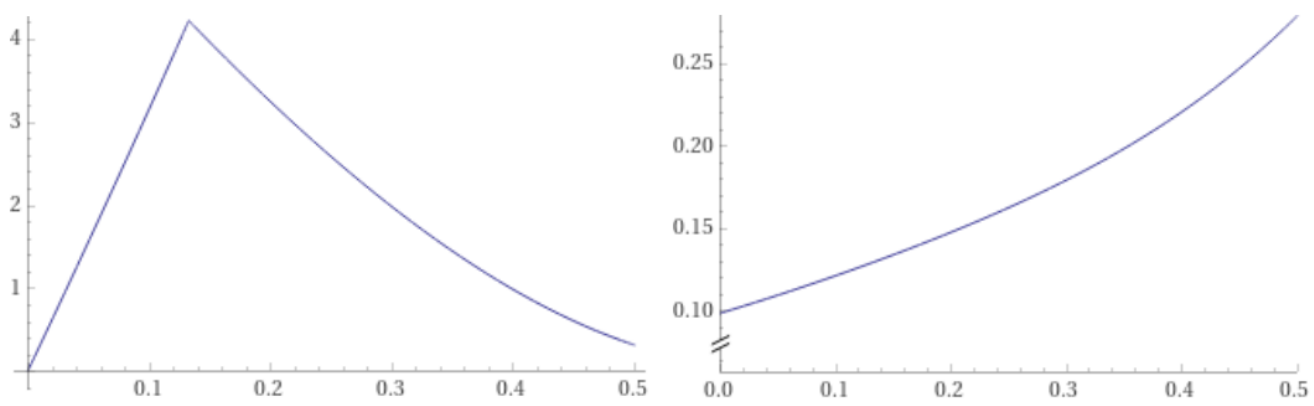

Figure 4. To illustrate the inequality (48), we have on the left $z_{1}(x)=\theta(x)-$ $\left|\varphi^{\prime \prime \prime}(x)+\frac{1}{6} x^{3} \varphi(x)+\frac{1}{5} x \varphi(x)-24 x-\int_{0}^{x} t \varphi(t)+\varphi(t) d t\right|$. On the right, we have $z_{2}(x)=$ $\frac{\alpha^{3}}{1-\left(\alpha^{3}+\frac{5}{4} \alpha^{4}\right)} \theta(x)-\left|\varphi_{0}(x)-\varphi(x)\right|$ with $\alpha=0.3095535$ to illustrate the inequality (49).

\subsection{Third Example: 3-Differentiable Function and a Bigger Perturbation}

We will consider the integro-differential Equation (44) but with another function $\theta$ and a bigger perturbation of the solution. Considering the continuous function $\theta:\left[0, \frac{1}{2}\right] \rightarrow$ $[0, \infty)$ defined by

$$
\theta(x)=6+0.3 x,
$$

we have

$$
\int_{0}^{x} 6+0.3 t d t \leq \alpha(0.6+0.3 x)=\alpha \theta(x)
$$

with $\alpha \in\left[\frac{81}{164}, \infty\right)$. 
Choosing $\varphi(x)=x^{3}+0.1$, it follows

$$
\begin{gathered}
\left|\varphi^{\prime \prime \prime}(x)+\frac{1}{6} x^{3} \varphi(x)+\frac{1}{5} x \varphi(x)-24 x-\int_{0}^{x} t \varphi(t)+\varphi(t) d t\right| \\
=\left|-\frac{1}{30} x^{6}-\frac{1}{20} x^{4}-\frac{1}{30} x^{3}-\frac{602}{25} x+6\right| \leq \theta(x),
\end{gathered}
$$

for all $x \in\left[0, \frac{1}{2}\right]$.

To illustrate the inequality (51), we have Figure 5.
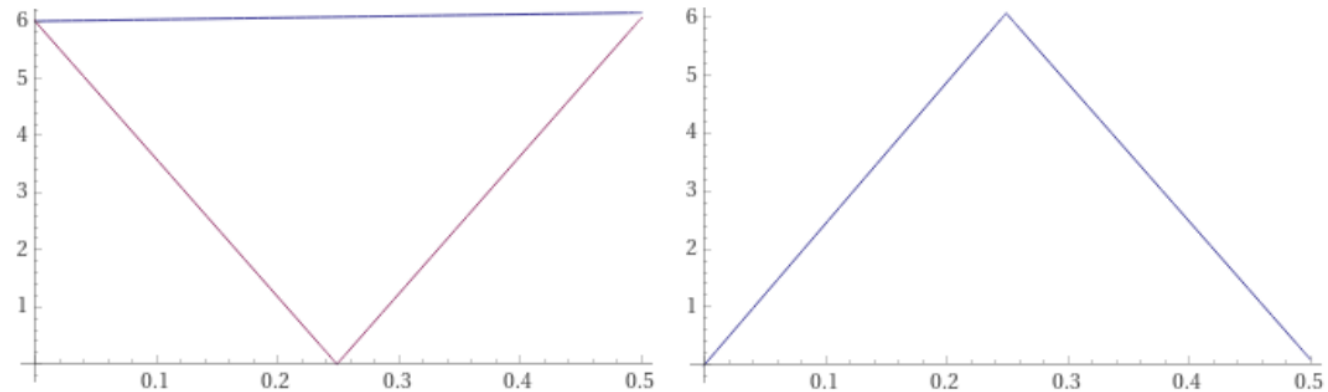

Figure 5. On the left we have $\theta(x)=6+0.3 x$ and $z_{1}(x)=\left|-\frac{1}{30} x^{6}-\frac{1}{20} x^{4}-\frac{1}{30} x^{3}-\frac{602}{25} x+6\right|$. On the right, we have $z_{2}(x)=\theta(x)-\left|\varphi^{\prime \prime \prime}(x)+\frac{1}{6} x^{3} \varphi(x)+\frac{1}{5} x \varphi(x)-24 x-\int_{0}^{x} t \varphi(t)+\varphi(t) d t\right|$.

Therefore, this result exhibits the Hyers-Ulam-Rassias stability of the integro-differential Equation (44).

Moreover, by using the exact solution $\varphi_{0}(x)=x^{4}$ with

$$
\alpha \in\left[\frac{81}{164}, 0.794596\right]
$$

we obtain $M\left(\alpha^{3}+L \alpha^{4}\right)<1$, and

$$
\left|\varphi_{0}(x)-\varphi(x)\right|=\left|x^{3}+0.1-x^{4}\right| \leq \frac{\alpha^{3}}{1-\left(\alpha^{3}+\frac{5}{4} \alpha^{4}\right)}(6+0.3 x)
$$

for all $x \in\left[0, \frac{1}{2}\right]$.

To illustrate the inequality (53), we have Figure 6.
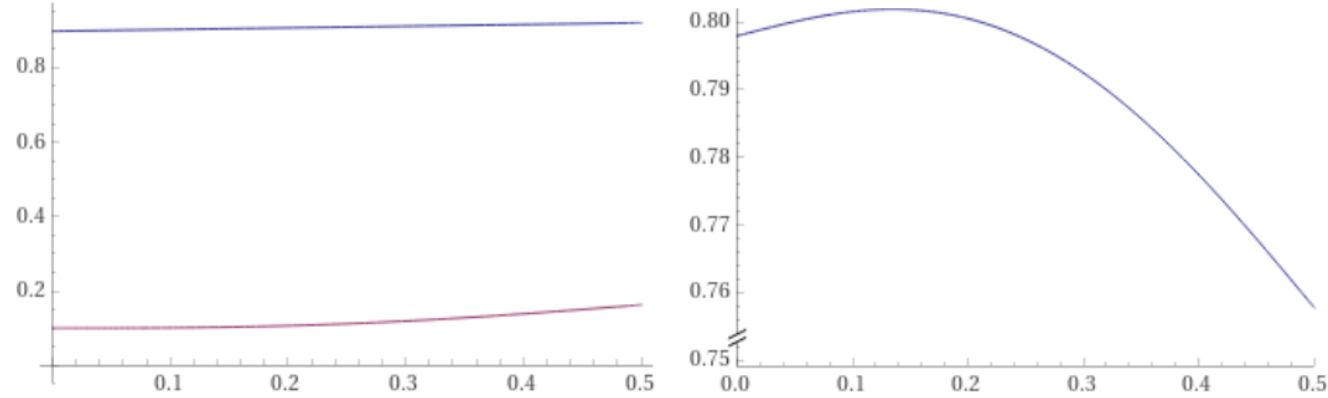

Figure 6. On the left, we have $z_{1}(x)=\frac{\alpha^{3}}{1-\left(\alpha^{3}+\frac{5}{4} \alpha^{4}\right)}(6+0.3 x)$ with $\alpha=0.49391$ and $z_{2}(x)=$ $\left|x^{3}+0.1-x^{4}\right|$. On the right, we have $z_{3}(x)=\frac{\alpha^{3}}{1-\left(\alpha^{3}+\frac{5}{4} \alpha^{4}\right)} \theta(x)-\left|\varphi_{0}(x)-\varphi(x)\right|$ with $\alpha=0.49391$.

\section{Conclusions}

In this work, we presented new sufficient conditions for the Hyers-Ulam-Rassias, the Hyers-Ulam and the $\sigma$-semi-Hyers-Ulam stabilities for a general higher order integrodifferential equation by using the Banach fixed point theorem and a generalization of the 
Bielecki metric thus enabling the study of the stability of an expanding number of particular equations. Some examples were presented to illustrate the theoretical results.

Author Contributions: Conceptualization, A.M.S., F.C. and P.C.; formal analysis, A.M.S., F.C. and P.C.; investigation, A.M.S., F.C. and P.C.; methodology, A.M.S., F.C. and P.C.; project administration, A.M.S.; resources, A.M.S.; supervision, A.M.S.; writing—original draft, A.M.S.; writing—reviw and editing, A.M.S., F.C. and P.C. All authors have read and agreed to the published version of the manuscript.

Funding: This work was partially supported by FCT-Portuguese Foundation for Science and Technology through the Center of Mathematics and Applications of University of Beira Interior (CMA-UBI), within project UIDB/00212/2020, the Center for Research and Development in Mathematics and Applications (CIDMA) of University of Aveiro, within project UIDB/04106/2020 and the Research Center for Mathematics and Applications (CIMA) of University of Évora, within project UIDB/04674/2020.

Institutional Review Board Statement: Not applicable.

Informed Consent Statement: Not applicable.

Data Availability Statement: Not applicable.

Conflicts of Interest: The authors declare no conflict of interest.

\section{References}

1. Ulam, S.M. A Collection of the Mathematical Problems; Interscience Publication: New York, NY, USA, 1960.

2. Hyers, D.H. On the stability of linear functional equation. Proc. Natl. Acad. Sci. USA 1941, 27, 222-224. [CrossRef] [PubMed]

3. Rassias, T.M. On the stability of the linear mapping in Banach spaces. Proc. Am. Math. Soc. 1978, 72, 297-300. [CrossRef]

4. Aoki, T. On the stability of the linear transformation in Banach spaces. J. Math. Soc. Jpn. 1950, 2, 64-66. [CrossRef]

5. Gajda, Z. On stability of additive mappings. Int. J. Math. Math. Sci. 1991, 14, 431-434. [CrossRef]

6. Rassias, T.M. On a modified Hyers-Ulam sequence. J. Math. Anal. Appl. 1991, 158, 106-113. [CrossRef]

7. Belluot, N.B.; Brzdęk, J.; Ciepliński, K. On some recent developments in Ulam's type stability. Abstr. Appl. Anal. 2012, $2012,41$.

8. Brzdęk, J.; Popa, D.; Rasa, I.; Xu, B. Ulam Stability of Operators; Elsevier Science Publishing Co Inc.: Amsterdam, The Netherlands, 2018.

9. Burton, T.A. Volterra Integral and Differential Equations, 2nd ed.; Mathematical in Science and Engineering; 202; Elseiver: Amsterdam, The Netherlands, 2005.

10. Corduneanu, C. Principles of Differential and Integral Equations, 2nd ed.; Chelsea: New York, NY, USA, 1988.

11. Gripenberg, G.; Londen, S.O.; Staffans, O. Volterra Integral and Functional Equations; Cambridge University Press: Cambridge, UK, 1990.

12. Lakshmikantham, V.; Rao, M.R.M. Theory of Integro-Differential Equations, Stability and Control: Theory, Methods and Applications 1; Gordon and Breach Publisher: Lausanne, Switzerland, 1995.

13. Alsulami, H.H.; Gülyaz, S.; Karapinar, E.; Erhan, I.M. An Ulam stability result on quasi-b-metric-like spaces. Open Math. 2016, 14, 1087-1103. [CrossRef]

14. András, S.Z.; Kolumbán, J.J. On the Ulam-Hyers stability of first order differential systems with nonlocal initial conditions. Nonlinear Anal. Theory Methods Appl. 2013, 82, 1-11. [CrossRef]

15. Bahyrycz, A.; Brzdęk, J.; Jablońska, E.; Malejki, R. Ulam's stability of a generalization of the Fréchet functional equation. J. Math. Anal. Appl. 2016, 442, 537-553. [CrossRef]

16. Brzdęk, J.; Popa, D.; Rassias, T.M. Ulam Type Stability; Springer International Publishing: Berlin/Heidelberg, Germany, 2019.

17. Buşe, C.; O'Regan, D.; Saierli, O.; Tabassum, A. Hyers-Ulam stability and discrete dichotomy for difference periodic systems. Bull. Des Sci. MathéMatiques 2016, 140, 908-934. [CrossRef]

18. Castro, L.P.; Simões, A.M. Hyers-Ulam and Hyers-Ulam-Rassias stability of a class of Hammerstein integral equations. In Proceedings of the AIP Conference Proceedings, ICNPAA 2016 WORLD CONGRESS: 11th International Conference on Mathematical Problems in Engineering, Aerospace and Sciences, La Rochelle, France, 4-8 July 2016; pp. 1-10.

19. Castro, L.P.; Simões, A.M. Hyers-Ulam-Rassias stability of nonlinear integral equations through the Bielecki metric. Math. Methods Appl. Sci. 2018, 1-17. [CrossRef]

20. Cho, Y.J.; Park, C.; Rassias, T.M.; Saadati, R. Stability of Functional Equations in Banach Algebras; Springer International Publishing: Berlin/Heidelberg, Germany, 2015.

21. Du, W.S. A generalization of Diaz-Margolis's fixed point theorem and its application to the stability of generalized Volterra integral equations. J. Inequal. Appl. 2015, 407, 1-15. [CrossRef]

22. Hassan, A.M.; Karapinar, E.; Alsulami, H.H. Ulam-Hyers stability for MKC mappings via fixed point theory. J. Funct. Spaces 2016, 2016, 11. [CrossRef]

23. Hyers, D.H.; Isac, G.; Rassias, T.M. Stability of Functional Equations in Several Variables; Birkhäuser: Basel, Swizerland, 1998. 
24. Jun, K.W.; Kim, H.M. Ulam stability problem for quadratic mappings of Euler-Lagrange. Nonlinear Anal. Theory Methods Appl. 2005, 61, 1093-1104. [CrossRef]

25. Jung, S.M. Hyers-Ulam-Rassias Stability of Functional Equations in Mathematical Analysis; Hadronic Press: Palm Harbor, FL, USA, 2001.

26. Park, D.W.; Lee, Y.H. The Hyers-Ulam-Rassias stability of the pexiderized equations. Nonlinear Anal. Theory Methods Appl. 2005, 63, 2503-2513. [CrossRef]

27. Popa, D.; Raşa, I. On the best constant in Hyers-Ulam stability of some positive linear operators. J. Math. Anal. Appl. 2014, 412, 103-108. [CrossRef]

28. Caulk, D.A.; Naghdi, P.M. Axisymmetric Motion of a Viscous Fluid Inside a Slender Surface of Revolution. J. Appl. Mech. 1987, 54, 190-196. [CrossRef]

29. Carapau, F.; Correia, P. Numerical simulations of a third-grade fluid flow on a tube through a contraction. Eur. J. Mech. B/Fluids 2017, 65, 45-53. [CrossRef]

30. Castro, L.P.; Simões, A.M. Different types of Hyers-Ulam-Rassias stabilities for a class of integro-differential equations. Filomat 2017, 31, 5379-5390. [CrossRef]

31. Brzdęk, J.; Cădariu, L.; Ciepliński, K. Fixed point theory and the Ulam stability. J. Funct. Spaces 2014, 2014, 16. [CrossRef]

32. Castro, L.P.; Guerra, R.C. Hyers-Ulam-Rassias stability of Volterra integral equations within weighted spaces. Lib. Math. 2013, 33, 21-35. [CrossRef]

33. Castro, L.P.; Ramos, A. Hyers-Ulam and Hyers-Ulam-Rassias stability of Volterra integral equations with delay. In Integral Methods in Science and Engineering; Constanda, C., Pérez, M., Eds.; Birkhäuser: Boston, MA, USA, 2010; pp. 85-94.

34. Castro, L.P.; Ramos, A. Hyers-Ulam-Rassias stability for a class of nonlinear Volterra integral equations. Banach J. Math. Anal. 2009, 3, 36-43. [CrossRef]

35. Castro, L.P.; Ramos, A. Hyers-Ulam stability for a class of Fredholm integral equations. In Mathematical Problems in Engineering Aerospace and Sciences ICNPAA 2010; Sivasundaram, S., Ed.; Cambridge Scientific Publishers: Cambridge, UK, 2010; pp. 171-176.

36. Castro, L.P.; Simões, A.M. Hyers-Ulam and Hyers-Ulam-Rassias stability of a class of integral equations on finite intervals. In Proceedings of the 17th International Conference on Computational and Mathematical Methods in Science and Engineering, Cádiz, Spain, 4-8 July 2017; pp. 507-515.

37. Castro, L.P.; Simões, A.M. Hyers-Ulam and Hyers-Ulam-Rassias stability for a class of integro-differential equations. In Mathematical Methods in Engineering: Theoretical Aspects; Tas, K., Baleanu, D., Machado, J.A.T., Eds.; Springer: Berlin/Heidelberg, Germany, 2019.

38. Castro, L.P.; Simões, A.M. Stabilities for a class of higher order integro-differential equations. AIP Conf. Proc. 2018, 2046. [CrossRef]

39. Cădariu, L.; Găvruţa, L.; Găvruça, P. Weighted space method for the stability of some nonlinear equations. Appl. Anal. Discrete Math. 2012, 6, 126-139. [CrossRef]

40. Tisdell, C.C.; Zaidi, A. Basic qualitative and quantitative results for solutions to nonlinear, dynamic equations on time scales with an application to economic modelling. Nonlinear Anal. 2008, 68, 3504-3524. [CrossRef] 